\title{
Editorial: ISAAC 2008 Special Issue
}

\author{
Seok-Hee Hong • Hiroshi Nagamochi
}

Received: 24 February 2011 / Accepted: 8 March 2011 / Published online: 17 March 2011

(C) Springer Science+Business Media, LLC 2011

This special issue contains a selection of six papers from the 19th Annual International Symposium on Algorithms and Computation (ISAAC 2008), which was held in Gold Coast, Australia on December 15-17, 2008. The ISAAC 2008 program committee accepted 78 papers among 229 high-quality submissions from 40 countries, after a rigorous review process. Among those accepted papers, the following six papers were selected and invited to this special issue, based on their evaluation by the program committee. These six papers all went through the standard refereeing process of Algorithmica before being accepted for publication.

The papers in this issue show recent advances in various areas of algorithms and theory of computation research. The first paper, "Signature Theory in Holographic Algorithms" by Cai and Lu, develops the signature theory in holographic algorithms, in terms of $d$-realizability and $d$-admissibility. For the class of 2-realizable signatures, they prove a Birkhoff-type theorem which determines this class. This is followed by characterization theorems for 1-realizability and 1-admissibility. Finally, they present some counting problems solvable in polynomial time by holographic algorithms.

The second paper, "Faster Parameterized Algorithms for MINIMUM FILL-IN" by Bodlaender, Heggernes and Villanger, presents two parameterized algorithms for the Minimum FILL-IN problem, also known as the CHORDAL COMPLETION problem. The first algorithm requires polynomial space, which improves the exponential part

S.-H. Hong (凶)

School of Information Technologies, University of Sydney, Sydney, NSW 2006, Australia

e-mail: shhong@it.usyd.edu.au

H. Nagamochi

Department of Applied Mathematics and Physics, Graduate School of Informatics, Kyoto University, Kyoto 606-8501, Japan

e-mail:nag@amp.i.kyoto-u.ac.jp 
of the previously known algorithm. The second algorithm improves the running time, at the cost of exponential space.

The third paper, "How to Guard a Graph?" by Fomin, Golovach, Hall, Mihalák, Vicari and Widmayer, studies the problem of finding the minimum number of cops needed to prevent a robber from entering the guarded region. They show that if the robber's region is a path, then the problem can be solved in polynomial time. However, the decision version of the problem is NP-complete when the robber moves in a tree. Furthermore, if the robber is moving in a directed acyclic graph, the problem becomes PSPACE-complete.

The fourth paper "The Complexity of König Subgraph Problems and AboveGuarantee Vertex Cover" by Mishra, Raman, Saurabh, Sikdar and Subramanian, studies the problem of finding König-Egerváry subgraphs of a given graph. They first study the algorithmic complexity of the ABOVE GUARANTEE VERTEX COVER problem. Then, they consider two variations of the König Subgraph problem, the König Vertex Deletion Problem and the Vertex (Edge) Induced König Subgraph Problem, and show that both problems are NP-complete. They also study the problems from the points of view of approximation and parameterized complexity.

The fifth paper, "A New Algorithm for Finding Trees with Many Leaves" by Kneis, Langer and Rossmanith, presents an algorithm that finds out-trees and outbranchings with at least $k$ leaves in directed graphs. These problems are known as Directed Maximum Leaf Out-Tree and Directed Maximum Leaf OutBRANCHIng, respectively, and as MAXIMUM LEAF SPANning TrEe for the case of undirected graphs. The algorithm runs faster and uses less space than the previously known algorithms for these problems.

The final paper, "An Efficient Scaling Algorithm for the Minimum Weight Bibranching Problem" by Babenko, presents a weight-scaling $O(m \sqrt{n} \log n \log (n W))$ time algorithm (where $W$ denotes the maximum magnitude of arc weights) for the minimum weight bibranching problem, assuming that arc weights are integers. A bibranching of a digraph generalizes both branchings and bipartite edge covers.

We wish to thank the authors for submitting their papers to the special issue. We also thank the referees for their thorough reviews and valuable comments that helped to improve many aspects of the papers published in this issue. Finally, we would like to thank the Editor-in-Chief, Ming-Yang Kao, for the opportunity to edit this special issue, and Melissa Fearon for her dedicated assistance. It is our hope that the papers in this issue will be a valuable resource for researchers and will stimulate further investigations into the many areas of algorithms and computation. 